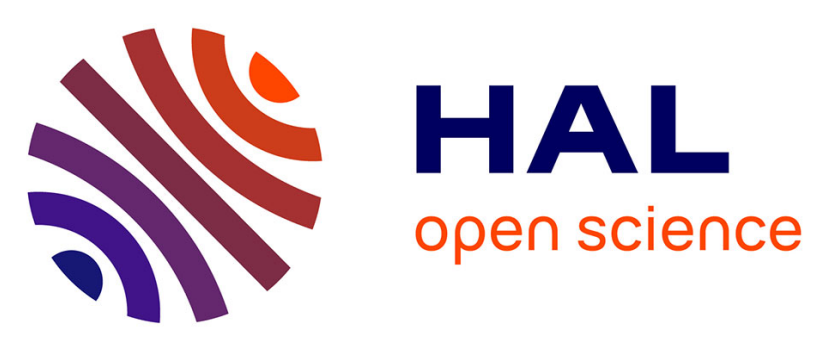

\title{
Oblate-Prolate Transition of Ellipsoidal Giant Magnetoliposomes: Experiments Showing an Anisotropic Spontaneous Curvature (Chapter 11)
}

Olivier Sandre, Christine Ménager, Jérôme Prost, Valérie Cabuil, Jean-Claude Bacri, Andrejs Cebers

\section{To cite this version:}

Olivier Sandre, Christine Ménager, Jérôme Prost, Valérie Cabuil, Jean-Claude Bacri, et al.. OblateProlate Transition of Ellipsoidal Giant Magnetoliposomes: Experiments Showing an Anisotropic Spontaneous Curvature (Chapter 11). Pier Luigi Luisi; Peter Walde. Perspectives in Supramolecular Chemistry, 6, , pp.169-180, 1999, Giant Vesicles, 9780470511534. 10.1002/9780470511534.ch11. hal-02320995

\section{HAL Id: hal-02320995 \\ https://hal.science/hal-02320995}

Submitted on 20 Oct 2019

HAL is a multi-disciplinary open access archive for the deposit and dissemination of scientific research documents, whether they are published or not. The documents may come from teaching and research institutions in France or abroad, or from public or private research centers.
L'archive ouverte pluridisciplinaire HAL, est destinée au dépôt et à la diffusion de documents scientifiques de niveau recherche, publiés ou non, émanant des établissements d'enseignement et de recherche français ou étrangers, des laboratoires publics ou privés. 


\title{
Oblate-Prolate Transition of Ellipsoidal Giant Magnetoliposomes: Experiments showing an Anisotropic Spontaneous Curvature
}

\author{
OLIVIER SANDRE ${ }^{1}$, CHRISTINE MENAGER ${ }^{2}$, JEROME PROST $^{2}$, VALERIE CABUIL $^{2}$, \\ JEAN-CLAUDE BACRI ${ }^{1}$, ANDREJS CEBERS ${ }^{1}$
}

${ }^{1}$ Lab. Milieux Désordonnés et Hétérogènes, Universités Paris 6 et Paris 7 (UMR 7603)

${ }^{2}$ Lab. Liquides Ioniques et Interfaces Chargées, Université Paris 6 (UMR 7612)

\section{Introduction}

The shape of fluid lipid vesicles is governed by the bending elasticity of their membrane characterized by a bending modulus $K_{c}$ and a spontaneous curvature $c_{0}$ [1]. The latter exists only for bilayers separating non symmetric aqueous media. Recently Döbereiner et al described an experimental method based on the thermal fluctuations of giant vesicles to measure $c_{0}$ precisely and use it as a control parameter in phase diagrams [2]. We use here particular non symmetric giant vesicles called 'magnetoliposomes' as a tool for the observation of new shape transitions. Magnetoliposomes are giant liposomes filled up with a colloidal dispersion of magnetic particles. As usual liposomes, magnetoliposomes exhibit thermal fluctuations. The effect of a magnetic field is to flatten the fluctuations and to induce a shape deformation even at low field intensity. Two types of deformations are reported here depending on the characteristics of the encapsulated magnetic solution. They are explained by a modification of $c_{0}$ induced by the application of the magnetic field.

\section{Liposome preparation}

Magnetoliposomes are obtained by filling usual giant liposomes with an aqueous magnetic fluid (ferrofluid). They are suspended in a non-magnetic aqueous phase. The ferrofluid is a colloidal suspension of magnetic nanoparticles (iron oxide grains $\gamma-\mathrm{Fe}_{2} \mathrm{O}_{3}$ ) [3]. Particle diameter is about $10 \mathrm{~nm}$ and the iron oxide surface is coated by tri-sodium citrate species in order to have negative surface charges at $\mathrm{pH} 7$, which ensure the colloidal stability even in physiological media [4]. There is an equilibrium between the citrate species adsorbed on the grains and the non-adsorbed tri-sodium citrate in the bulk. This electrolyte has a concentration $C_{S}$ which is monitored by dialysis and controlled by measuring the conductivity of the ferrofluid. The phospholipid constituting the membrane of the magnetoliposomes is 1,2-dioleoyl-sn-glycero-3-phosphocholine (DOPC from Avanti Polar Lipids, USA, used as purchased). 
For the preparation of giant liposomes, we use the spontaneous swelling of a dense phospholipidic film because it enables to encapsulate a ferrofluid inside giant vesicles with a high efficiency. The ferrofluid is firstly prepared and its ionic strength $C_{s}$ is adjusted by dialysis. A small amount of lipid powder $(1 \mathrm{mg})$ is mixed with $10 \mu \mathrm{L}$ of this aqueous ferrofluid ( $\mathrm{pH}=7$, volume fraction in magnetic particles is 0.05$)$ and sheared on a glass support to obtain a dense lamellar phase. Then tridistilled water is added in excess $(4 \mathrm{~mL})$ to allow the spontaneous swelling of the liposomes during one hour incubation at $40^{\circ} \mathrm{C}$. Most of the liposomes prepared by this way are spherical with diameter ranging from 10 to $100 \mu \mathrm{m}$ (Fig. 1). They are red colored and they exhibit thermal fluctuations.

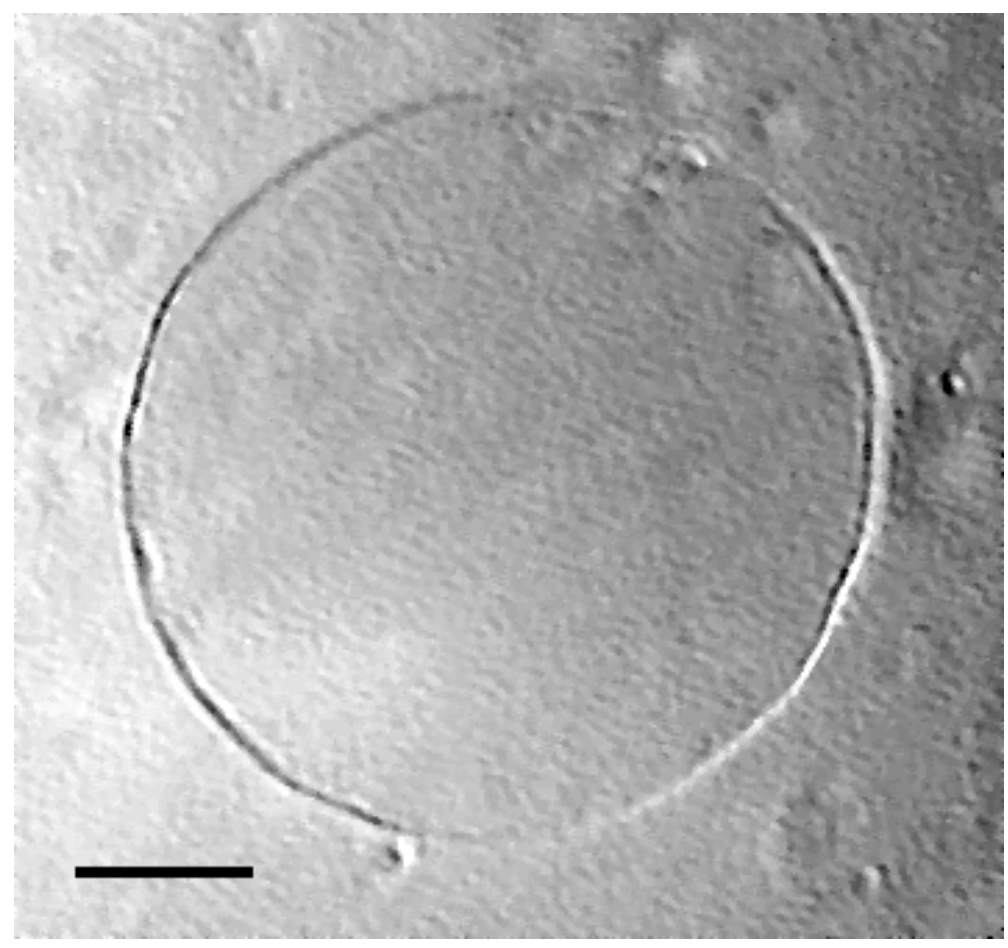

Fig. 1: A giant magnetoliposome without any magnetic field. The membrane is fluctuating as observed with differential interference contrast microscopy (DIC). Length of the bar is $20 \mu \mathrm{m}$.

\section{Observations}

Cells made of two glass slides separated by a $200 \mu \mathrm{m}$ spacer are filled by aliquots of the magnetoliposomes suspension and observed under an optical microscope (Leica $\times 40$, NA 0.65). Pictures are taken with a CCD camera and digitized on a computer.

The magnetoliposomes are sensitive to an applied magnetic field of low intensity, typically 400 G. Two types of deformations have been observed. An initially spherical magnetoliposome is either elongated by the field (Fig. 2) or on the contrary compressed at the poles (Fig. 3). The shape is always described by an ellipsoid with rotational symmetry along the field direction: $a$ is the semi-axis parallel to the magnetic field $\vec{H}$ and $b$ is the value of the two other semi-axes perpendicular to $\vec{H}$. 


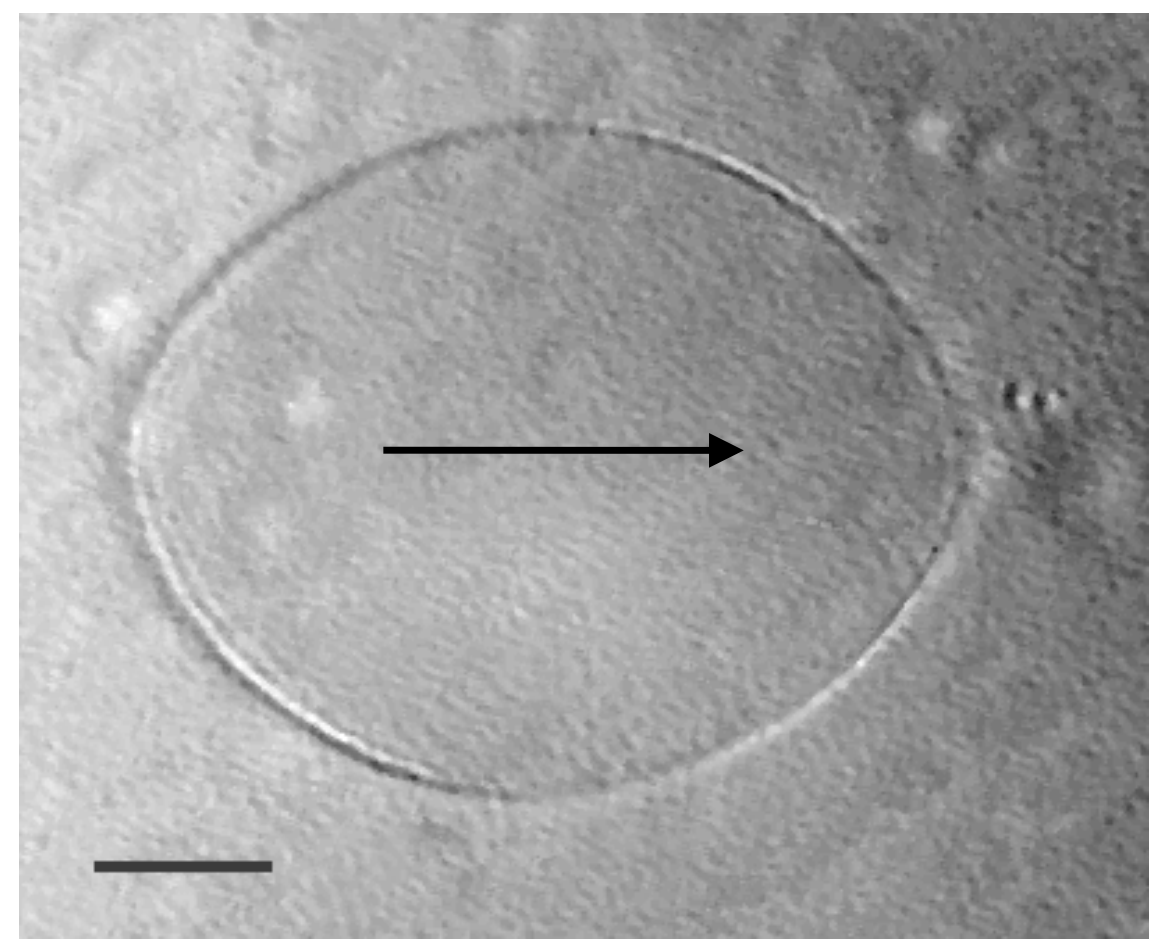

Fig. 2: The liposome shown in Fig.1 submitted to a magnetic field (direction given by the arrow). The deformation is prolate and the membrane fluctuations vanish. Please note that the ionic strength is high $(C s=85 \mathrm{mM})$. Length of the bar is $20 \mu \mathrm{m}$.

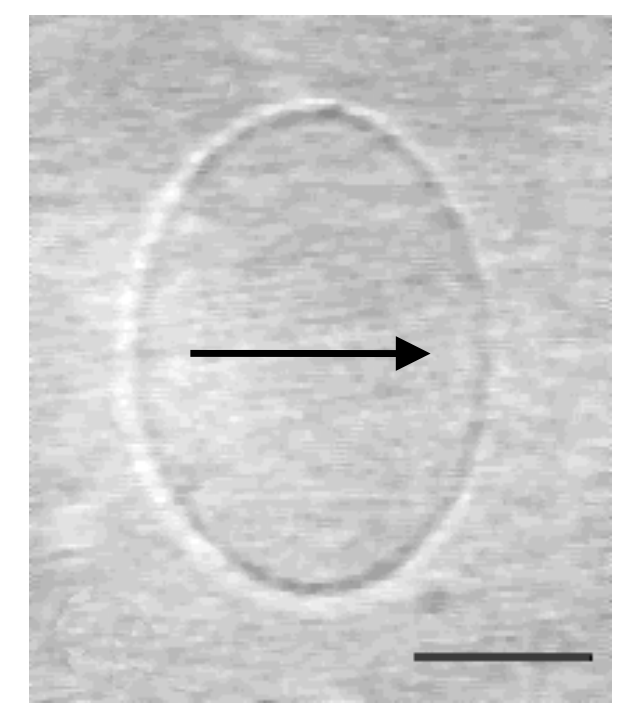

Fig. 3: A magnetoliposome filled with a low ionic strength magnetic fluid ( $C s=7.75$ $\mathrm{mM}$ ) and submitted to a magnetic field (direction given by the arrow). The deformation is oblate. Length of the bar is $10 \mu \mathrm{m}$.

The type of deformation and its amplitude are described by the ellipsoid eccentricity: $e^{2}=1-\frac{b^{2}}{a^{2}}$. For an elongated liposome (prolate ellipsoid), $e^{2}$ is positive, and for a compressed one (oblate ellipsoid), $e^{2}$ is negative. A combination of the two deformations is also observed and described as a 'spinning top' shape (Fig. 4). 
Author manuscript version of Chapter 11 published in Perspectives in Supramolecular Chemistry Vol 6: Giant

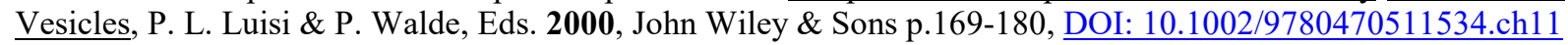

a)
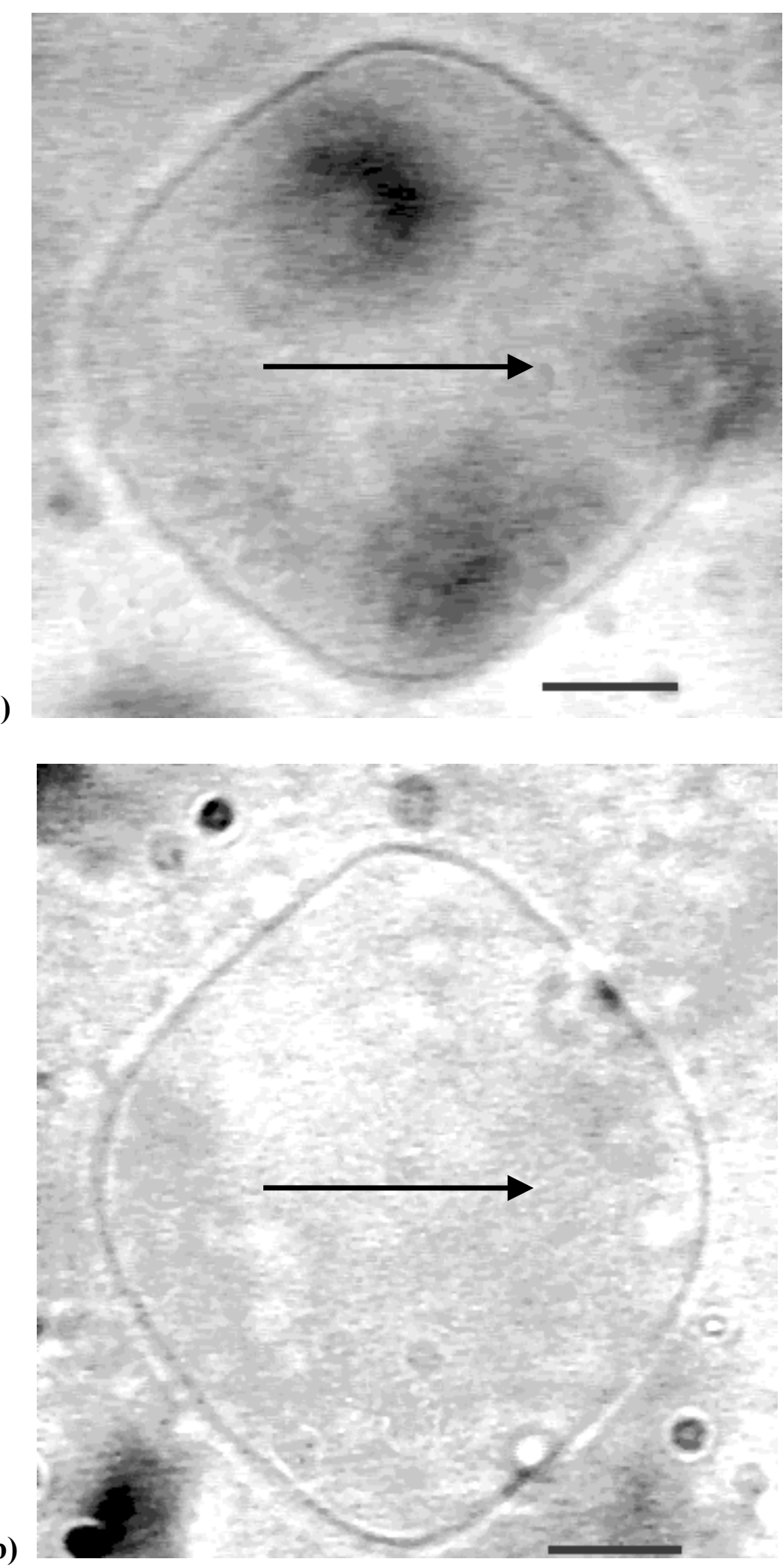
c)

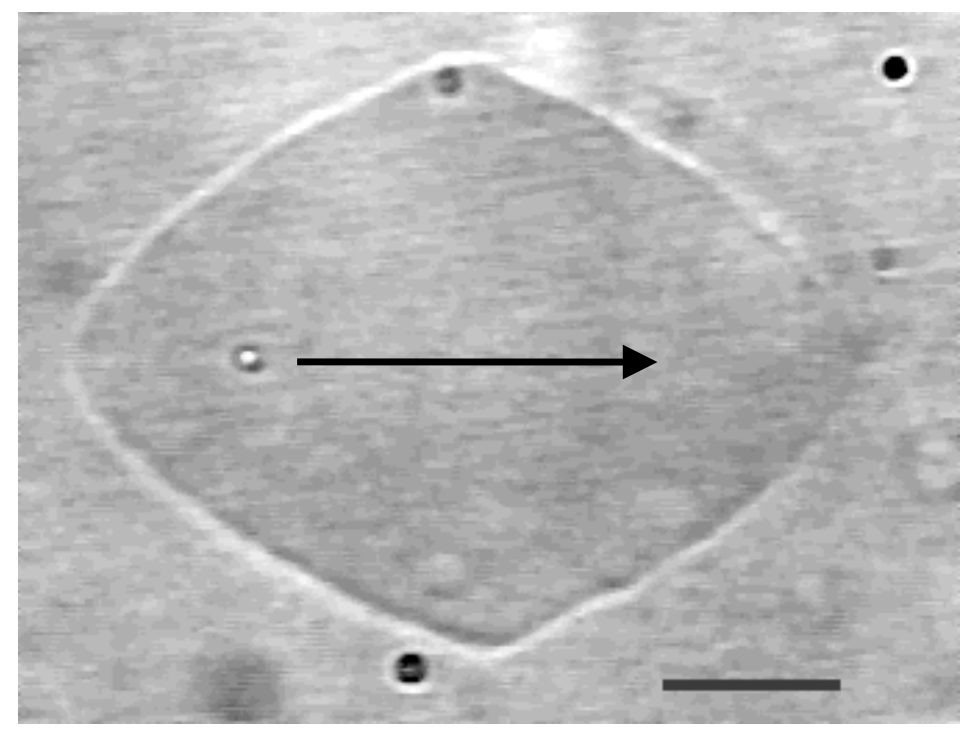

d)

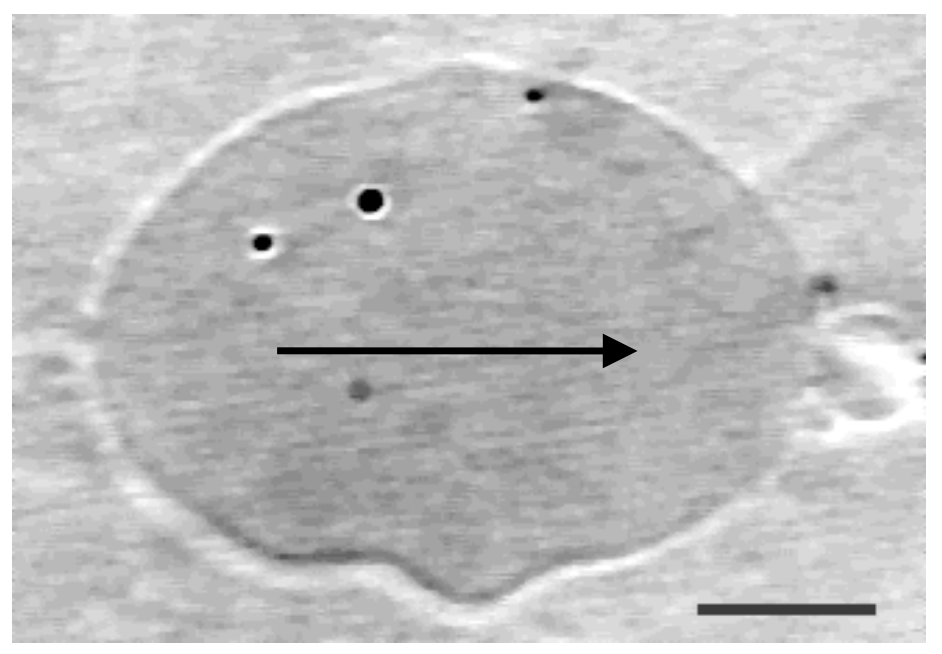

Fig. 4 (a to d): Magnetoliposomes inside which salt concentration is intermediate $(C s=25 \mathrm{mM})$ and submitted to a magnetic field (direction given by the arrow). The liposomes are always axisymmetric around the field direction but combine elongations both at the poles and at the equator ('spinning top' shapes). The values of can be nearly zero (a), negative (b) or positive (c). In that last case the sharp shape is only transient and relaxes to a more rounded one, but with membrane buckling $(\mathrm{d})$. Length of the bar is always $10 \mu \mathrm{m}$.

\section{Analysis of the deformations under a magnetic field}

The experimental parameters that are relevant for the observed shape transitions are (a) the initial radius $R_{0}$ of the liposome measured on the video frame and (b) the two characteristics of the encapsulated solution, namely its magnetic susceptibility $\chi$ and the free salt concentration $C_{S}$.

An in situ measurement of $\chi$ is provided by magnetophoresis, i.e. the migration of a liposome submitted to a controlled gradient of magnetic field. A magnetic field gradient is performed perpendicularly to an applied magnetic field with the set-up described in Fig. 5. 


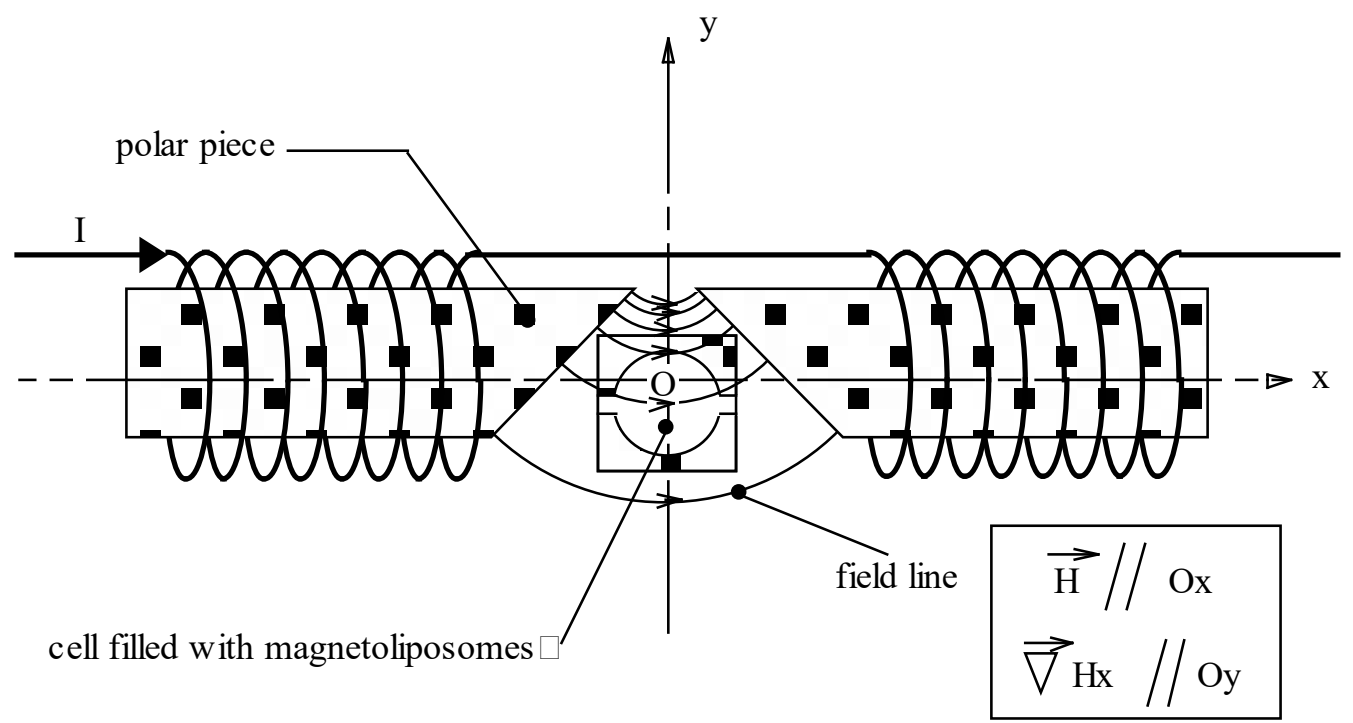

Fig. 5: Experimental set-up to produce a controlled magnetic field gradient (the field lines get closer toward increasing $y$ coordinate). The sample containing the magnetoliposomes is placed between the two coils in the cell described in the text.

The field lines of two coils in serial are deviated by polar pieces edged at $45^{\circ}$. The sample is in the middle region where the field lines are parallel to the $\mathrm{O}-\mathrm{x}$ axis and the field intensity gradient is in the $\mathrm{O}-\mathrm{y}$ direction. The gradient is $110 \pm 2 \mathrm{G} \cdot \mathrm{cm}^{-1}$ (measured with Hall effect probes) with a field equal to $410 \pm 2 \mathrm{G}$ in the sample place.

The magnetic force which causes the migration of a magnetoliposome submitted to a magnetic field gradient is:

$$
\vec{F}_{m}=\chi V H_{x}\left(\vec{\nabla}_{y} H_{x}\right)
$$

where $V=\frac{4}{3} \pi a b^{2}$ is the volume of the liposome and $\vec{\nabla}$ is the gradient operator. It is balanced by a viscous force, which was calculated by J. Perrin [5] in the case of an ellipsoid elongated in the $\mathrm{O}-\mathrm{x}$ direction and moving along $\mathrm{O}-\mathrm{y}$ in a medium of viscosity $\eta$ :

$$
\vec{F}_{v}=16 \pi \eta \frac{a}{2 \ln \left(\frac{2 a}{b}\right)+1} \vec{v}
$$

The viscosity of the suspending solution of the vesicles is $\eta=10^{-2} \mathrm{P}$. The magnetophoretic velocity results from the balance of the two forces:

$$
\vec{v}=\frac{b^{2}\left\lfloor 2 \ln \left(\frac{2 a}{b}\right)+1\right\rfloor}{8 \eta} \chi H_{x}\left(\vec{\nabla}_{y} H_{x}\right)
$$

The measurement of the liposome velocity leads to the value of $\chi$ characterizing the ferrofluid encapsulated in a given liposome. The concentration $C_{P}$ of nanoparticles inside the liposome is then calculated using Langevin's law of superparamagnetism :

$$
\chi=\frac{C_{p} V_{p}^{2} m_{s}^{2}}{3 k T}
$$


where $m_{s}=360 \mathrm{G}$ is the magnetization at saturation of $\gamma-\mathrm{Fe}_{2} \mathrm{O}_{3}$ and $V_{P}$ is the volume of a nanoparticle [6]. In the following we use $V_{P}$ corresponding to a particle diameter of $12 \mathrm{~nm}$, taking into account that the ferrofluid is polydisperse and that $\chi$ is ruled by the tail of the distribution.

Please note that $R_{0}$ and $C_{P}$ vary a lot among the population of liposomes due to the swelling process. Their values are measured thereafter. The concentration of tri-sodium citrate salt in the ferrofluid is adjusted to a known value by dialysis through a cellulose membrane (Spectra/Por MWCO 12-14000 $\mathrm{g} \cdot \mathrm{mol}^{-1}$ from ROTH, France). Final concentrations $C_{S}$ ranging from $5 \mathrm{mM}$ to $85 \mathrm{mM}$ are measured from the electrical conductivity of the ferrofluid.

The experimental values of $R_{0}$ and $\chi$ are measured on a large amount of magnetoliposomes prepared with ferrofluids of different $C_{S}$. Some of them are given as examples in Table 1. On Fig. 6 experimental data illustrate the influence of the three parameters $R_{0}, \chi$ and $C_{S}$ on the observed deformations. Vesicles which are larger, more magnetic and filled with a high ionic strength magnetic fluid exhibit prolate deformation under a magnetic field, whereas smaller vesicles filled with a low ionic strength magnetic fluid and less magnetic become oblate. Two domains corresponding to both types of deformation are well defined on this 'topological' phase diagram, the oblate one being in the region of low values of $R_{0}, \chi$, and $C_{S}$.

\begin{tabular}{|c|c|c|c|c|}
\hline$C_{S}\left(\mathrm{~mol} \cdot \mathrm{L}^{-1}\right)$ & $\chi$ & $C_{P}\left(\mathrm{~cm}^{-3}\right)$ & $R_{0}(\mu \mathrm{m})$ & $e^{2}=1-\frac{b^{2}}{a^{2}}$ \\
\hline $5 \times 10^{-3}$ & $7.1 \times 10^{-5}$ & $8.2 \times 10^{13}$ & 25.2 & -0.397 \\
\hline $7.75 \times 10^{-3}$ & $1.1 \times 10^{-4}$ & $1.3 \times 10^{14}$ & 13.3 & -0.596 \\
\hline $7.75 \times 10^{-3}$ & $7.5 \times 10^{-5}$ & $8.7 \times 10^{13}$ & 21.4 & -1.17 \\
\hline $2.5 \times 10^{-2}$ & $7.3 \times 10^{-5}$ & $8.4 \times 10^{13}$ & 24.6 & -0.845 \\
\hline $2.5 \times 10^{-2}$ & $1.2 \times 10^{-4}$ & $1.4 \times 10^{14}$ & 22.5 & 0.496 \\
\hline $2.5 \times 10^{-2}$ & $6.5 \times 10^{-5}$ & $7.6 \times 10^{13}$ & 50.4 & 0.343 \\
\hline $3.5 \times 10^{-2}$ & $3.6 \times 10^{-5}$ & $4.2 \times 10^{13}$ & 21.1 & -1.31 \\
\hline $4.5 \times 10^{-2}$ & $5.5 \times 10^{-5}$ & $6.4 \times 10^{13}$ & 31.6 & 0.466 \\
\hline $6.5 \times 10^{-2}$ & $1.1 \times 10^{-3}$ & $1.2 \times 10^{15}$ & 14.1 & 0.536 \\
\hline $8.5 \times 10^{-2}$ & $1.4 \times 10^{-4}$ & $1.7 \times 10^{13}$ & 13.0 & 0.586 \\
\hline
\end{tabular}

Table 1: Examples of experimental data about magnetoliposomes. $C_{S}$ is the concentration of tri-sodium citrate electrolyte in the ferrofluid, $R_{0}$ the radius of the initial spherical shape, $e$ the eccentricity of the ellipsoidal deformation and $\chi$ the magnetic susceptibility. The concentration $C_{p}$ of magnetic particles in situ is calculated from $\chi$ using Eq. 4. The shadowed lines correspond to prolate deformations $\left(e^{2}>0\right)$, the other to negative ones $\left(e^{2}<0\right)$. 


\section{Interpretation}

When submitted to an applied magnetic field, a liposome encapsulating a ferrofluid of susceptibility $\chi$ gets a bulk magnetization (proportional to $R_{0}^{3}$ ); as a consequence, the interface between inner magnetic and outer non-magnetic media (i.e. the bilayer) is stressed. The deformation of an initially spherical object by a magnetic stress should always be a prolate ellipsoid, whatever the sign of $\chi$, so that magnetic droplets and magnetic holes (eg. air bubbles) have the same behavior [7]. This was observed for magnetoliposomes and used in [8] in order to determine the bending modulus $K_{c}$ of the bilayer from the analysis of the liposomes deformation as a function of the magnetic field intensity.

Therefore the observation of an oblate deformation by a slight change of the experimental parameters is a tricky result. Please note that the inner compartment of the magnetoliposomes is a complex colloidal solution. It includes both a $3: 1$ electrolyte (trisodium citrate) with concentration $C_{s}$ and a colloidal suspension of nanometric $\gamma-\mathrm{Fe}_{2} \mathrm{O}_{3}$ particles (giving $\chi$ ). The latter are both nanoscopic magnets and macro-ions, the net charge per nanoparticle being $Z \approx-20$ [4]. The importance of decreasing $C_{S}$ to obtain an oblate deformation suggests that there are electrostatic interactions between the particles and the bilayer. Indeed some electrophoresis experiments on large unilamellar liposomes (diameters about $200 \mathrm{~nm}$ ) made of phospholipids with a phosphocholine head group have reported values of the $\zeta$-potential ranging from $-4 \mathrm{mV}$ for natural egg-lecithin [9] to about $-10 \mathrm{mV}$ for synthetic lipids like 1,2-dimysristoyl-sn-glycero-3-phosphocholine (DMPC) [10]. The $\zeta-$ potential varies with temperature, ionic strength and specific adsorption of anions [11]. We retain for the present study that the bilayer and the magnetic nanoparticles are both negatively charged and so repel each other.

The shape of magnetoliposomes submitted to a magnetic field results from a competition between the magnetic energy of the bulk and the bending energy of the bilayer. The magnetic energy always favors the prolate shape, but we shall show that the bending energy can stabilize the oblate deformation. The contribution to the bending energy of the bilayer due to a surface charge density has been extensively studied theoretically $[12,13,14]$, but to our knowledge only experiments on small unilamellar vesicles (diameters about $10 \mathrm{~nm}$ ) have been reported [15]. The shape of liposomes is known to be strongly dependent on the spontaneous curvature of the bilayer $\left(c_{0}\right)$. In the case of magnetoliposomes, $c_{0}$ originates from the asymmetry between the aqueous compartments (ferrofluid inside, non-magnetic solution outside). Winterhalter and Helfrich [12] have introduced a method to derive the elasticity constants of a bilayer bearing two different charge densities on its both sides. If we use the same approach, considering that the screening length of the electrostatic potential is $\kappa_{i}^{-1}$ inside the liposome and $\kappa_{o}^{-1}$ outside, the spontaneous curvature can be written:

$$
c_{0}=\frac{\kappa_{o}^{3}}{3}\left(\frac{1}{\kappa_{o}^{2}}-\frac{1}{\kappa_{i}^{2}}\right)
$$


The effect of the magnetic field (intensity $H_{0}$ ) on the spontaneous curvature $c_{o}$ is due to the modification it implies on the particles concentration profile in the vicinity of the bilayer. Qualitatively the depletion in nanoparticles is reduced near the magnetic poles because of magnetic attraction. The effect of this phenomenon on $c_{0}$ can be quantitatively described introducing an angular dependence in the expression of the decay factor of the electrostatic potential away from the bilayer:

$$
\kappa_{i}^{2}(\theta)=\kappa_{o}^{2}\left|1+\frac{d}{1+\frac{4 \pi \chi^{2} H_{0}^{2}}{C_{p} k T} \cos ^{2}(\theta)}\right|
$$

where $\theta$ is the angle between the field direction and the normal to the bilayer and $d$ is the ratio $\frac{Z^{2} C_{p}}{12 C_{s}}$ [16]. Thus, when a magnetic field is applied, $c_{0}$ becomes lower near the poles $(\theta=0$ and $\left.\theta=180^{\circ}\right)$ than at the equator. But the angular contribution of $\kappa_{i}$ according to Eq. 6 depends on the amount of magnetic materials through $C_{P}$ and $\chi$, and on the ionic strength through $d$. This explains the driving role of these parameters in the experiments (Fig. 6).

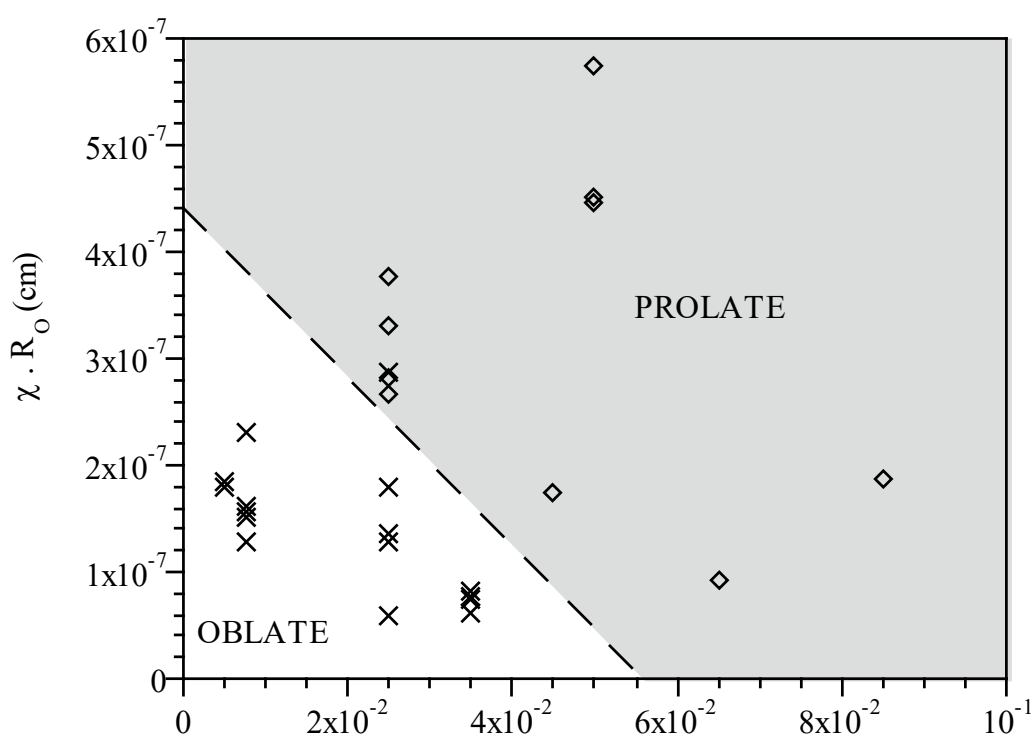

a)

Electrolyte concentration $\mathrm{C}_{\mathrm{s}}$ (mol citrate ions per Liter)

b)

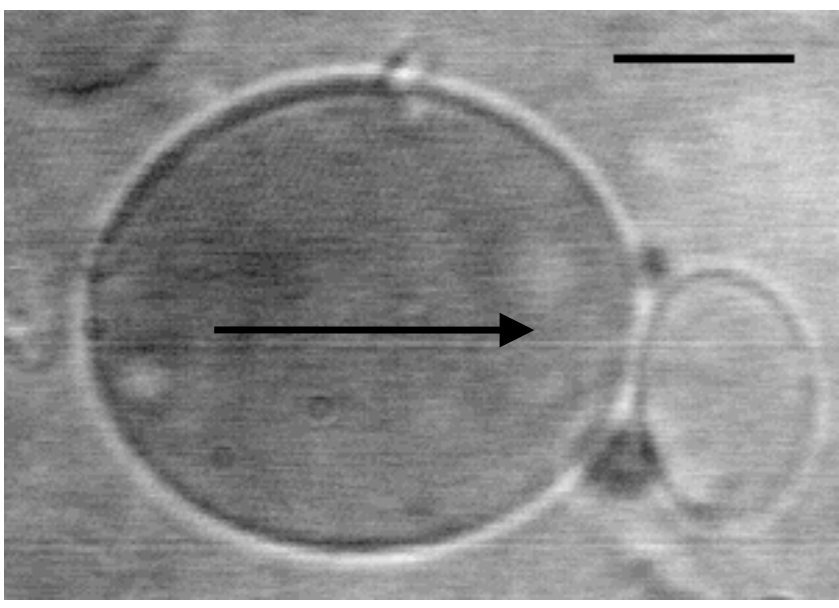


Fig. 6: (a) 'Topological' phase diagram of the magnetoliposomes under a magnetic field where product $\chi R_{0}$ is plotted versus tri-sodium citrate salt concentration $C_{S}$ in the ferrofluid. The dotted line between the two domains of prolate and oblate deformations is only a guide for the eye; (b) Effect of product $\chi R_{0}$ at given salt concentration $\left(C_{S}=25 \mathrm{mM}\right)$ on two neighboring liposomes with opposite deformations under a magnetic field. The prolate one is large and highly colored (high $\chi R_{0}$ ) while the oblate one is smaller and appears more dilute in magnetic particles (low $\chi R_{0}$ ). Length of the bar is $10 \mu \mathrm{m}$. The arrow indicates the direction of the field.

For example a decrease of $C_{S}$ (by dialysis of the ferrofluid) raises up the anisotropy of the spontaneous curvature. Hence the oblate shape minimizes the bending energy because it develops a less curved bilayer at the magnetic poles where $c_{0}$ is decreased. If the concentration of magnetic oxide is not too high, so that the bending energy prevails over the magnetic one, then the oblate shape is favored. This explains the tendency of oblate deformation under a magnetic field for magnetoliposomes encapsulating a ferrofluid diluted both in electrolyte and in nanoparticles.

We have described in this article a new shape transition of magnetoliposomes. We interpret this experimental result by a coupling between magnetostatics and electrostatics. A complete theoretical model will be proposed in a forthcoming paper. The main point of our results at the present time is that even a small charge density on the phospholipidic membrane can induce a coupling with charged encapsulated species.

\section{References}

[1] Helfrich W (1973) Z Naturforsch 28c : 693-703.

[2] Döberheiner HG, Evans E, Kraus M, Seifert U Wortis M (1997) Phys Rev E 55 : 44584474.

[3] Massart R (1981) IEEE Trans Magn MAG 17: 1247-1248.

[4] Bacri JC, Perzinski R, Salin D, Cabuil V, Massart R (1990) J Magn Magn Mat 85: 27-32.

[5] Perrin J (1936) J. Phys. Radium 7: 33.

[6] Bacri JC, Salin D, Perzinski R, Cabuil V, Massart R (1986) J Magn Magn Mat 62: 36-46.

[7] Skjeltorp AT (1987) J Magn Magn Mat 65 : 195; Skjeltorp AT (1983) Phys Rev Lett 51:

2306; Skjeltorp AT (1984) J Appl Phys 55: 2587.

[8] Bacri JC, Cabuil V, Cebers A, Ménager C, Perzynski R (1996) Europhys Lett 33: 235-240.

[9] Carrion FJ, De La Maza A, Parra JL (1994) J Colloid Interf Sci 164: 78-87.

[10] Makino K, Yamada T, Kimura M, Oka T (1991) Biophys Chem 41: 175-183.

[11] Tatulian SA (1983) Biochim Biophys Acta 736: 189-195.

[12] Winterhalter M, Helfrich W (1988) J Phys Chem 92: 6865-6867 ; Winterhalter M, Helfrich W (1992) J Phys Chem 96: 327-330.

[13] Pincus P, Joanny JF, Andelman D (1990) Europhys Lett: 763-768.

[14] Bensimon D, David S, Leibler S, Pumir A (1990) J Phys France 51: 689-695.

[15] Oberdisse J, Porte G (1997) Phys Rev E 56: 1965-1975.

[16] Sandre O, Ménager C, Prost J, Cabuil V, Bacri J-C, Cebers A (2000) Phys Rev E, 62: 3865-3870. 